Techniques \& Culture

\title{
L'animal de zoo
}

Un rôle entre sauvage et domestique

\section{Garry Marvin}

\section{(2) OpenEdition}

Journals

Édition électronique

URL : https://journals.openedition.org/tc/3944

DOI : $10.4000 /$ tc.3944

ISSN : 1952-420X

Éditeur

Éditions de l'EHESS

\section{Édition imprimée}

Date de publication : 31 décembre 2008

Pagination : 102-119

ISSN : 0248-6016

Référence électronique

Garry Marvin, «L'animal de zoo », Techniques \& Culture [En ligne], 50 | 2008, mis en ligne le 31 décembre 2010, consulté le 29 septembre 2022. URL : http://journals.openedition.org/tc/3944 ; DOI https://doi.org/10.4000/tc.3944 


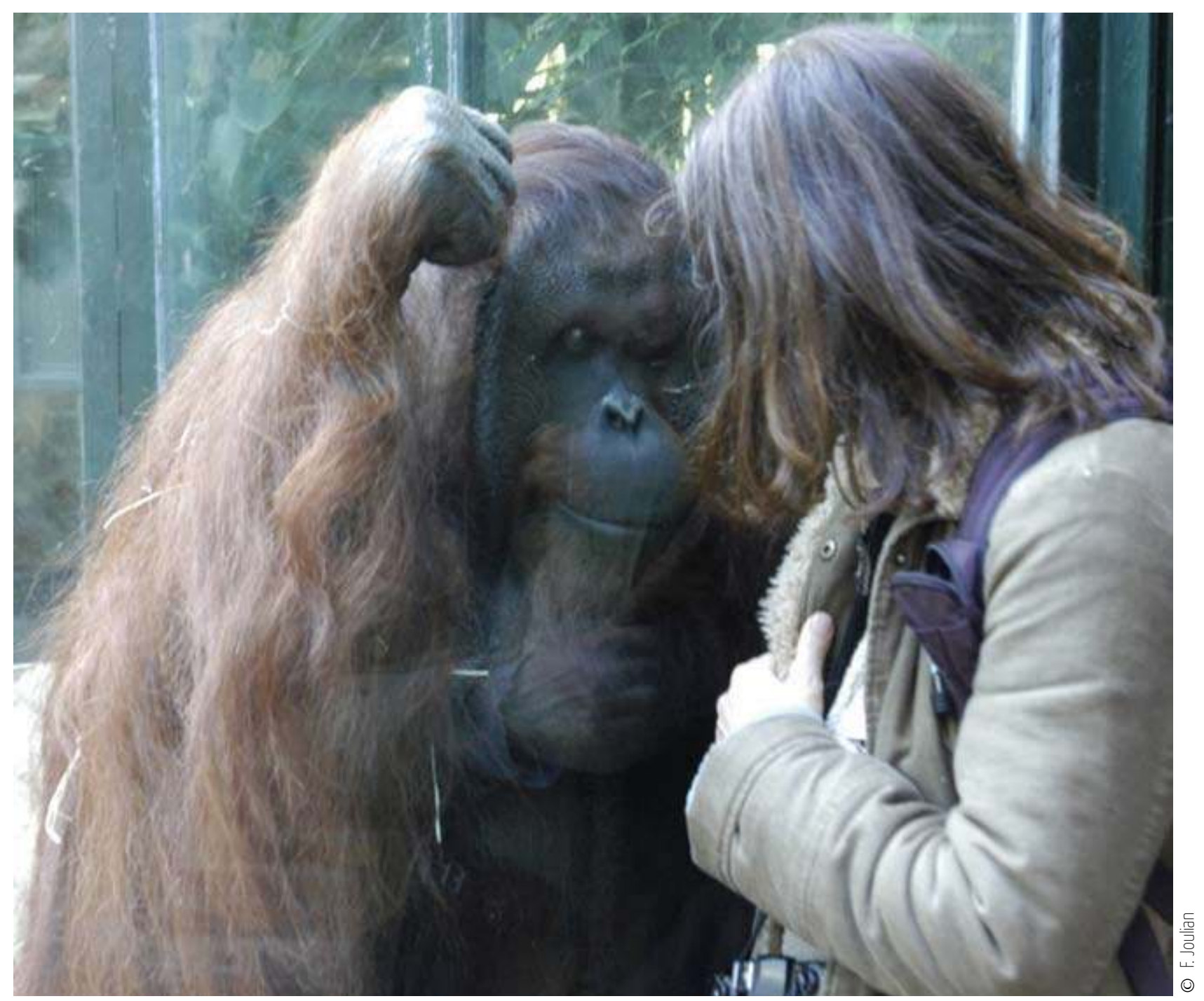




\title{
L'ANIMAL DE ZOO
}

\section{Un rôle entre sauvage et domestique}

\author{
L'auteur envisage ici le zoo comme un décor de théâtre destiné à la production \\ de discours et d'imaginaire, dans lequel l'animal de zoo - animal liminal, \\ entre sauvage et domestique - entraîne le visiteur dans l'ailleurs d'un monde \\ sauvage fictif.
}

Les zoos sont peuplés, peut-être même habités, par des milliers d'animaux singuliers représentant une impressionnante diversité d'espèces. Leur désignation et leur classification en tant qu'« animal sauvage », tant dans le discours ordinaire que dans la littérature sur les zoos, leur assignent, à un certain niveau, une identité partagée ${ }^{1}$. Cette identité diffère par bien des aspects de celle d' ' animal domestique ${ }^{2}$. Or, il me semble que l'application de la catégorie « sauvage » aux animaux qui habitent les zoos est problématique. En conséquence, j’aimerais sonder la possibilité de leur appartenance à un autre registre d'identité collective, que l'on pourrait appeler « animal de zoo ». Ces animaux, malgré leurs nombreuses différences, ont en commun un certain nombre d'attributs particuliers, en raison de l'endroit où ils vivent, de leur mode de vie, ainsi que de leur exposition continue à la présence humaine. Les conceptions et les classifications de l'animal « sauvage » ou « domestique » varient selon les sociétés et les cultures, mais les animaux sont généralement - quoique non exclusivement - assignés à ces catégories en fonction de la perception de ce qu'est une identité spécifique. L'« animal de zoo » relève d'un autre régime de classification et d'identité, 
fondé sur le rôle qui lui est assigné et qu'il joue à l'intérieur du dispositif du zoo, plutôt que sur la perception de l'identité de son espèce.

Mon intérêt, qui va au-delà du constat de la simple existence de « l'animal de zoo » pour se focaliser sur les aspects pragmatiques de sa construction dans l'imaginaire humain, découle d'une attention plus générale portée aux processus et aux pratiques de représentation des animaux. Dans un numéro spécial de la revue Society and Animals, consacré à la question de la représentation, Steve Baker remarque:

«"Lampleur de la désillusion quant à la possibilité de représenter le réel" (Berterns 1995 : 11) comme caractéristique du monde postmoderne a été démontrée de façon convaincante. Pourtant, beaucoup reste à dire sur le thème démodé et non-postmoderne du « réel », et sur le savoir qui est considéré comme connaissance de l'animal dans le monde contemporain » (Baker 2001 : 195).

Cette désillusion ne me perturbe pas. Nous ne parviendrons jamais à découvrir l'animal « réel », bien que ce qu'en pensent et ce qu'en disent ceux qui se lancent dans cette entreprise mérite la plus grande attention. Les scientifiques, par exemple, qui travaillent dans des disciplines comme la biologie, la zoologie, l'éthologie ou l'écologie, peuvent bien prétendre étudier les animaux « réels » plutôt que leurs représentations culturelles, leurs travaux n'en sont pas moins socialement et culturellement circonscrits. Ce n'est pas mon propos que de remettre en cause l'existence d'animaux menant une vie autonome dans le monde, d'êtres dont l'identité singulière ne dépend pas de l'attention que leur portent les humains, mais je me demande bien comment nous pourrions les rencontrer, interagir avec eux et les comprendre sans la médiation de la culture. L'ambition de synthétiser les questionnements sur les animaux du point de vue du constructivisme social ou culturel peut paraître démesurée, mais c'est le fil que j'aimerais suivre dans cet article ${ }^{3}$. J'adopterai donc la posture d'un agnostique concernant la notion d'animal « réel » et ses natures possibles, afin de comprendre comment les animaux dans leur ensemble relèvent d'une construction des hommes - parfois au sens littéral -, mais sans jamais perdre de vue les relations qu'ils entretiennent avec eux et l'imagination dont ils sont l'objet. Ici, la notion de représentation est fondamentale. J'ai déjà eu l'occasion de postuler l'existence d'un rapport entre « présenter » et « représenter » à propos des animaux:

« La notion de "présentation" peut être comprise comme une référence à une façon élémentaire d'être au monde: directe, immédiate, immanente, désignant des animaux présents par et pour eux-mêmes. Ladjonction du préfixe "re" implique au contraire la présence obligatoire d'un autre être, actif et agissant celui-là - un agent ayant ses idées propres à propos de l'essence de ce qui est présent. La notion de présentation est en quelque sorte subordonnée à celle de représentation; celle-là relève d'un autre registre, intrinsèquement baigné de signification culturelle. Ce parti implique une série de questions spécifiques: qui est engagé dans la représentation? Représentation de quoi? Pour qui? À qui? À quelles fins et dans quels contextes? » (Marvin 2001 : 275-276). 
Les représentations ont des conséquences pour les animaux, en ce qu'elles structurent leurs rencontres avec les hommes.

Certes, ces rencontres sont différentes en fonction des divers animaux et publics dans les zoos, mais je voudrais m’arrêter plus spécifiquement ici sur ce qui caractérise tous ces animaux en tant que membres de la catégorie distincte d'animal de zoo. Ma proposition est la suivante: l'animal de zoo est un animal « liminal ». Si les animaux du zoo sont présents et habitent effectivement cet espace, la manière dont ils sont imaginés en fait également des représentants et des représentations d'animaux venus d'ailleurs, plus précisément de l'espace auquel ils appartiennent « naturellement ». Lanimal de zoo présente la particularité d'être en transit sans avoir l'objectif de se rendre où que ce soit. Les animaux de zoo sont, d'une certaine manière, une anomalie, et à bien des égards ambigus. Ils sont clairement apparentés aux animaux sauvages, mais je cherche à comprendre comment ils sont représentés en tant que tels et comment les zoos les représentent.

\section{Les acteurs du zoo}

Nous parlons d'animaux sauvages par opposition aux animaux domestiques. Un animal sauvage vit indépendamment et séparément des humains, il n'est pas directement sous leur contrôle et n'a pas été soumis au processus complexe de la domestication, il ne dépend pas directement des humains pour son existence, il prend généralement la fuite lorsque ceux-ci s'approchent de trop près, il est d'une certaine façon libre de ses mouvements et de sa vie. Sa reproduction, tout comme sa nourriture et ses relations sociales sont de son ressort et on imagine, en général, que les animaux sauvages habitent de grands espaces ouverts, " naturels », des contrées lointaines ou de la campagne. Ces généralités sont simplistes: les animaux sauvages peuvent dépendre de la protection que les humains leur accordent, par exemple en leur réservant des espaces dits « naturels». On en trouve en milieu urbain également: dans la rue où j'habite il y a de nombreux renards, on rencontre des écureuils dans la plupart des jardins, au bout de la rue on peut apercevoir des rats et des souris filer en tous sens dans le métro, et les oiseaux sont partout. Nombre de ces animaux subsistent grâce aux humains, étant dépendants par exemple de la consommation de leurs déchets alimentaires ou des commodités offertes par leur habitat. Mais nous continuons néanmoins à les concevoir comme des animaux sauvages. Il faut bien admettre la persistance d'une distance, qui se manifeste de différentes manières et qui semble déterminante dans notre façon de percevoir ou de catégoriser un animal comme sauvage.

Les animaux domestiques sont créés pour servir les intérêts humains et n'existeraient pas sans eux. Ils dépendent entièrement des hommes et leur sont associés de près. Ils vivent dans une relation complexe et constante de proximité. Les humains décident où et comment ils vont vivre, ce qu'ils vont manger, quand ils vont se reproduire et quand ils 


\section{Liminaire}

Lièvre patagon, jardin zoologique de BuenosAires, 2005.

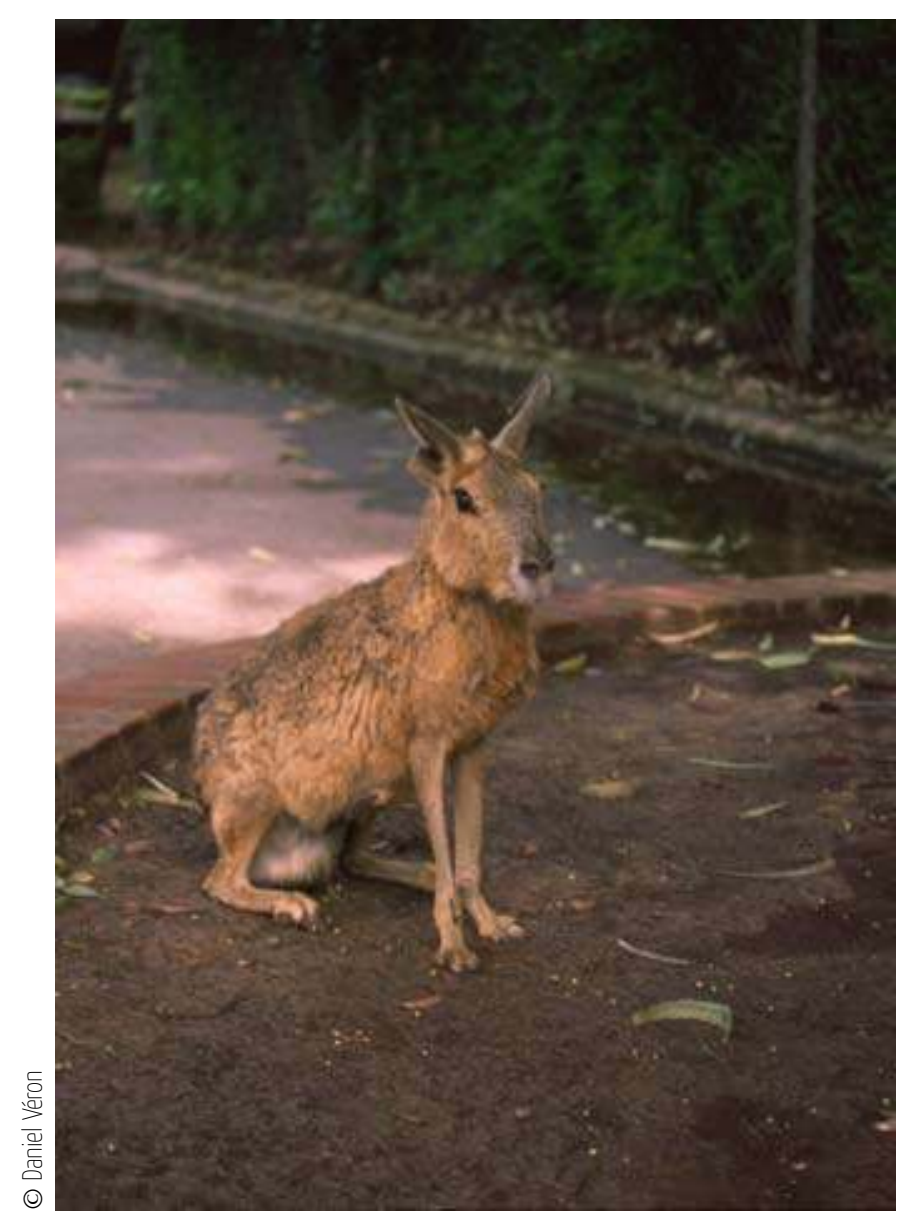

vont mourir. Ils les contrôlent, les soignent, et parfois les invitent même dans leur maison à partager leur vie de famille ${ }^{4}$.

Les animaux de zoo catégorisés comme « sauvages » - par opposition à « domestiques »-semblent être des créatures ambiguës et il m'est difficile d'évaluer combien ils sont sauvages ou comment cela transparaît dans leur comportement. Ils n'ont pas été domestiqués de la manière où nous l'entendons pour le bétail, les chevaux, les chèvres, les moutons, les cochons, les chiens et les chats, mais sont pourtant attentivement et intensivement pris en charge par les humains qui leur fournissent leur espace vital, leur nourriture et leur eau, qui les lavent, qui les soignent lorsqu'ils sont malades et qui décident de leur statut reproductif 5 . Mais bien qu'ayant vécu en zoo pendant des générations sous le contrôle direct des humains, ces créatures sont toujours perçues comme des animaux sauvages plutôt que domestiques. L'existence de différentes expressions pour désigner l'acte de garder un animal, qui s'applique aussi bien aux animaux de zoo qu'aux animaux domestiques, est éloquente à l'égard de cette ambivalence. Au zoo, les animaux sont gardés en captivité, tandis que les animaux domestiques sont tout simplement gardés; jamais les animaux domestiques ne sont considérés comme étant captifs (à l'exception peut-être dans le langage des défenseurs de la cause animale) bien qu'ils soient incapables d'aller nulle part ailleurs. La notion de captivité semble impliquer une connotation d'immoralité dans le fait de garder des animaux sauvages; les individus de ces espèces ne sont pas à leur juste place ou à leur place naturelle. Peut-être est-il vrai que d'autres membres de l'espèce sont effectivement dans un ailleurs qui leur est moralement ou naturellement dévolu, tandis que les animaux sauvages capturés dans leur milieu naturel puis amenés au zoo sont en captivité. Mais que penser des créatures nées et élevées au zoo par des parents eux-mêmes nés et élevés au zoo? Dans quelle mesure peut-on considérer qu'ils vivent en captivité plutôt que dans leur environnement d'origine, alors que le zoo est le seul environnement 
qu'ils aient jamais connu? Captifs, ils le sont au sens où ils ne peuvent pas partir. Mais les chevaux, les moutons, le bétail, les cochons, les chats et les chiens domestiques peuvent-ils décider de quitter l'endroit ou ils ont été élevés pour aller vivre ailleurs? C'est ce qui fait tout l'intérêt de la question du rôle donné à l'animal de zoo : il est à la fois ici (présent) et d'ici (il vit au zoo - il n'en sortira jamais) mais il est également là-bas (distant, absent et seulement imaginé), sa vie est rattachée à un ailleurs. Il fait référence à deux espaces en même temps, mais ne vit que dans un seul. Il doit représenter son homologue sauvage tout en restant l'individu qu'il est. En ce sens, il s'agit d'un animal acteur jouant le rôle d'un animal sauvage qui n'est pas présent, ou qui est présent ailleurs uniquement.

L'idée du zoo lui-même espace de représentation sera l'objet d'une analyse plus approfondie dans les pages qui suivent, mais il convient avant tout de souligner qu'il est lui aussi doté des propriétés d'ici et d'ailleurs. Il est tout à la fois de l'endroit où il se trouve, et en provenance de lieux situés bien au-delà de son enceinte. Ainsi, la volonté des zoos modernes (occidentaux) de nier leur existence propre - le fait qu'ils sont ici - dans leurs façons de présenter et de représenter les animaux n'est pas anodine. Globalement, les zoos ne content pas ce que sont leurs animaux et comment ils y vivent, mais bien des histoires à propos de contrées lointaines. Le cadre des enclos et leurs décors évoquent l'Asie,

Hippopotames

Jardin zoologique de Buenos-Aires, 2005.

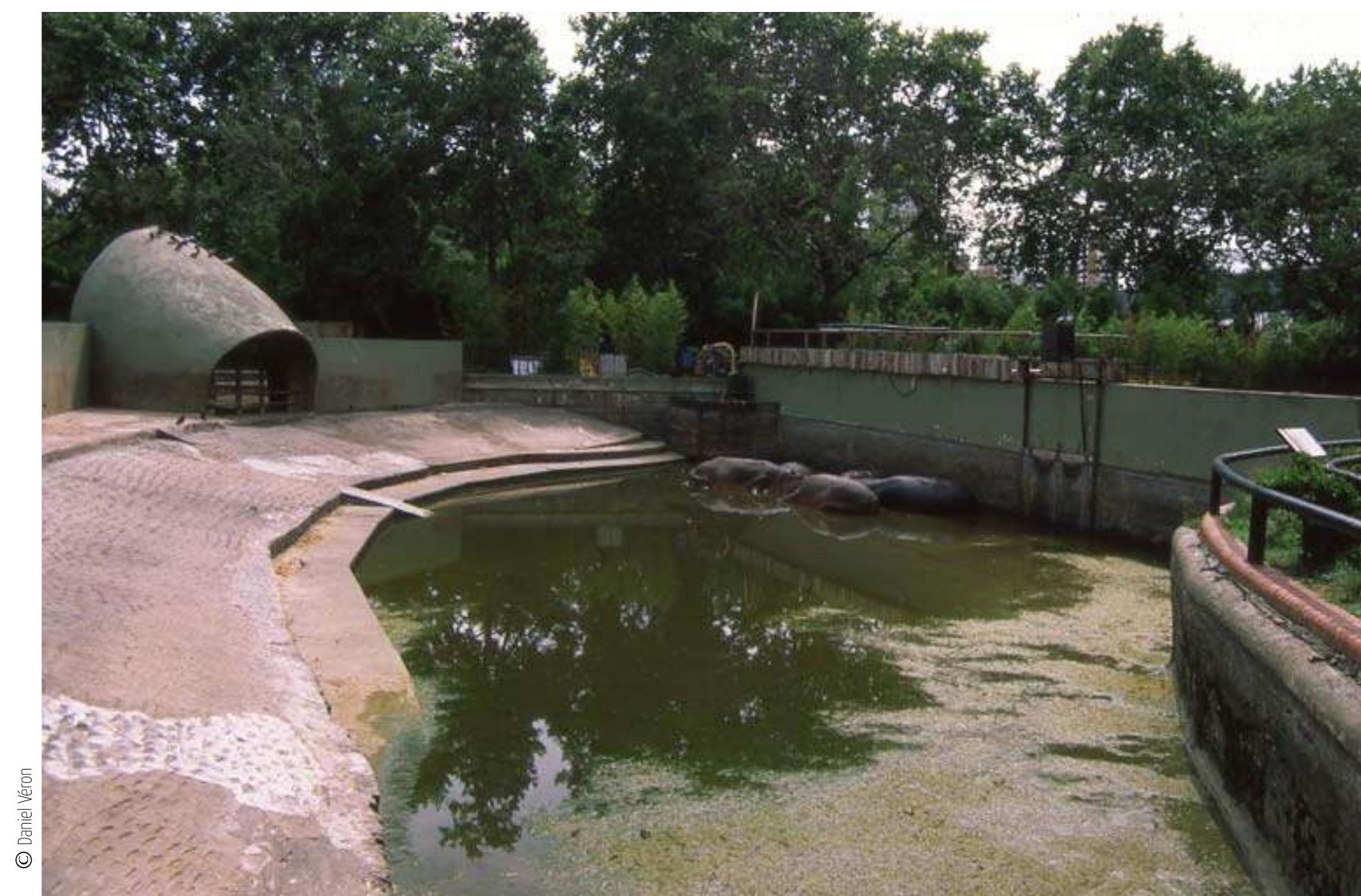


l'Afrique ou l'Amérique du Nord, en évacuant toute référence à un espace où l'on garde des animaux sauvages. À cet égard, l'observation des informations que les zoos diffusent à propos de la localisation de leurs animaux, par exemple, est fascinante. «Felis leo - le lion d'Afrique - vit dans les vastes régions de l'Afrique de l'Est» ou «Ursus maritimus - l'ours polaire - dans les régions arctiques de l'Amérique du Nord». Les panneaux se gardent bien de dire que tel animal « habite cet endroit dans le centre de Paris, de Berlin ou de Londres », bien que ce lion ou cet ours polaire se trouve effectivement au même endroit que les visiteurs qui le regardent. Même si cela peut sembler trivial, je crois qu'il faut s'intéresser aux manières dont chaque individu au zoo est destiné à représenter d'autres membres de son espèce. À mon avis, il s'agit là d'un problème fondamental tant pour ceux qui sont chargés de l'exposition et de la philosophie de présentation des zoos, que pour le type de rencontre qu'y font humains et animaux. Le contact (essentiellement par la vue) avec l'animal ou les animaux qui s'offrent à notre regard est direct et immédiat, mais la plupart des zoos s'en servent en vue de promouvoir une rencontre imaginée avec des animaux qui ne sont pas présents, et dont le mode de vie est très différent de la vie des animaux telle qu'elle est réellement vécue au zoo.

Ici, la référence au principe de «naturalisation » semble particulièrement importante. Dans ce contexte, la naturalisation peut être définie comme le processus par lequel les membres d'une espèce, importés de leur environnement naturel, se maintiennent en tant qu'espèce dans un nouvel environnement. Comme l'écrivent Eric Baratay et Elisabeth Hardouin-Fugier, il est question de « naturalisation », ou d'« adaptation à un nouvel environnement tout en restant à l'état sauvage » (2002 : 142). Mais l'« état sauvage » des animaux de zoo est quelque peu particulier. Ces animaux ont bien la morphologie physique des animaux sauvages - contrairement aux programmes de reproduction des animaux domestiques, ceux des zoos n'ont pas pour objectif de modifier la morphologie de l'animal - et en ce sens les zoos tentent de préserver une authenticité du corps de l'animal sauvage. Toutefois, le respect de l'expression du caractère sauvage, qui correspond aux normes comportementales de l'espèce, semble bien plus problématique et limité. Non seulement l'environnement est créé à l'intention des animaux par des architectes et autres designers, mais ils sont rarement maintenus en groupes sociaux spontanés et ils ne peuvent pas se reproduire de façon naturelle. Ils interagissent rarement avec d'autres espèces, ils ne sont pas capables de tuer d'autres animaux et sont eux-mêmes protégés contre tout danger de mort. En conséquence, le dilemme des zoos est le suivant: ils content une histoire sur la vie de la faune qui ne peut se jouer dans le décor du zoo. Le discours sur la vie de la faune sauvage est voué à rester un discours, une fiction, sans jamais devenir cette vie: les conditions du zoo ne permettent pas à la faune de mener une vie pleinement sauvage, quelle que soit l'ampleur des moyens consentis pour encourager les animaux à l'imiter. 


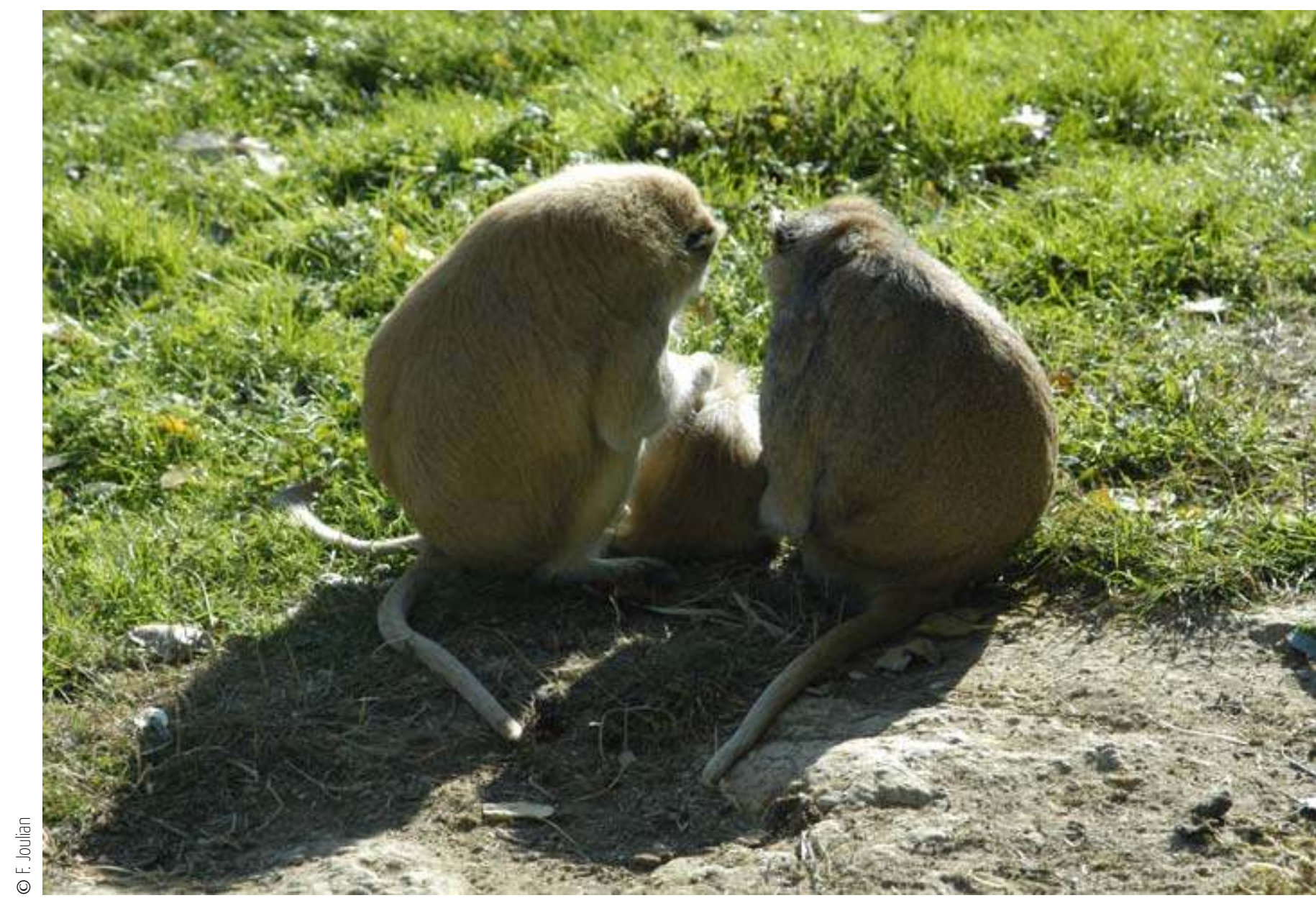

Primates vus de dos

La visibilité est également un paramètre fondamental qui entre dans la construction de "l'animal de zoo », le distinguant de "l'animal sauvage ». Le fait qu'il soit visible en permanence est une des principales exigences du zoo. Or la possibilité d'échapper au regard humain et la liberté de refuser une relation avec les hommes qui cherchent leur contact comptent parmi les facultés des animaux sauvages vivant en milieu naturel. Celui qui cherche à les observer doit déjouer leurs sens - la vue, l'odorat, l'ouie - afin de rester invisible et de n'être pas détecté. L'observateur humain doit prendre la posture d'un animal afin de tenter de comprendre comment les animaux pratiquent leur environnement, il doit s'y immerger et n'être pas vu pour voir. C'est dans ce type de milieu et par ce mode d'observation que les animaux sauvages peuvent être eux-mêmes. Dans la nature, les humains qui cherchent à observer les animaux ne s'annoncent pas et ne révèlent pas leur présence, à l'inverse de ce qui se produit dans les zoos.

Zooparc de Beauval, 2002. 
De nombreux sens sont mis à contribution au zoo - l'odorat, l'ouie et occasionnellement le toucher -, mais c'est la vue qui est privilégiée. Le langage du zoo est visuel: spectacle, décor, exposition. Les animaux ont peu d'occasions d'échapper à l'observation publique, voyeuriste, sans-gêne et intrusive des visiteurs. Nombre d'entre eux peuvent sentir qu'ils sont observés, sont conscients d'être regardés ou perçoivent par d'autres sens une présence à laquelle ils ne peuvent se soustraire ou échapper. Ils ne peuvent fuir le regard des humains ${ }^{6}$. Ma thèse est que cette visibilité forcée et continue contribue au moins pour partie à forger l'animal de zoo, tout comme l'inutilité partielle de ses sens. En milieu naturel, les animaux en ont besoin pour survivre - pour savoir s'il y a des prédateurs ou des proies à proximité, pour entendre les bruits qui indiquent un danger, pour sentir l'odeur d'autres créatures qu'ils veulent débusquer ou éviter. Leurs sens leur permettent, entre autres, d'entrer ou non en relation avec les autres animaux et avec le monde qui les entoure. Dans les zoos, nombre de ces sens, n'ayant pas d'opportunité naturelle d'expression ou de raison d'être, perdent leur utilité : l'antilope n'a pas besoin de craindre la vue, l'odeur ou le bruit du lion. Aucun animal n'a à s'orienter puisqu'ils n'ont nulle part où aller. Leurs sens se déplacent donc vers d'autres usages et s'orientent particulièrement vers leurs relations avec les humains. Ces relations sont au centre de leur attention alors que celles avec leurs congénères sont minimales et les interactions avec d'autres espèces sont rares. Dès leur naissance, les animaux sont habitués à vivre en présence des humains et cette présence dicte et contrôle l'expression de leur manière d'être.

L'individualité des animaux sauvages dans les zoos mérite également que l'on s'y arrête un instant. En milieu naturel, ils sont perçus comme des représentants de leur espèce et en conséquence sont rarement reconnus ou assimilés par les humains à des individus. Mais en zoo, leur nombre limité et le resserrement du cadre de l'attention conduit à la reconnaissance de nombre d'entre eux en tant que tels (ceci semble dépendre de l'espèce et concerner avant tout les membres de certaines grandes espèces de mammifères). Ce phénomène est accentué par la pratique de l'octroi d'un nom individuel, propre à chaque animal, dans de nombreux zoos. Le choix des noms est lui aussi très intéressant, en particulier les noms « exotiques» qui semblent lier l'animal à la culture des hommes de sa prétendue région d'origine. Aux États-Unis ou au Royaume Uni par exemple, les éléphants d'Afrique sont souvent affublés d'un nom typiquement « africain », comme Omari, Indula ou M'Dundella. Les pandas géants se voient souvent imposer un nom selon le même schéma, par exemple Mei Xiang, Tian Tian, Shi Shi ou Hua Mei. Si les animaux dans la nature n'ont aucun nom (à l'exception de ceux qui sont étudiés par les zoologistes de terrain - un cas très intéressant), ceux des zoos, qui en reçoivent un, sont ainsi personnalisés et introduits dans l'arène des intérêts humains. Quels animaux sont nommés? Quels sont ceux qui peuvent recevoir un nom? Quels noms leur donne-t-on? Les réponses à ces questions sont révélatrices des divisions internes et des spécificités de l'animal de zoo. 
Avant de clore cette partie, je voudrais revenir brièvement sur les notions de sauvage et de domestique et soulever la question du but de la vie animale et des raisons de son existence, sans la poser d'un point de vue métaphysique cependant. Si l'on admet que les animaux ont une raison d'être qui leur est propre - se maintenir, se reproduire, exprimer et accomplir le propre de leur espèce -, les animaux domestiques, en revanche, ont à peine la possibilité d'avoir une vie significative, qui soit la leur et qu'ils vivent pour eux-mêmes. Leur existence est vouée à satisfaire des besoins humains; c'est leur raison d'être et celle pour laquelle ils sont entretenus. En ce sens, les animaux de zoo ont beaucoup en commun avec les animaux domestiques et sont très différents de leurs homologues sauvages. Les raisons de leur existence ne sont pas les mêmes que celles des animaux domestiques, mais s'ils relèvent d'une autre classe d'intérêts, leur raison d'être n'en est pas moins dictée par le commerce des hommes. Elle tient en partie à leur visibilité, aux divertissements qu'ils procurent, à leur présence au centre des relations et des expériences humaines. Cette situation peut sans doute être envisagée en termes de rôles: dans les pages suivantes, je l'aborderai en termes de langage théâtral. Si l'on peut dire, de ce point de vue, que les animaux sauvages ont un rôle (biologiquement défini), les animaux domestiques et les animaux de zoo ont alors des rôles très différents, qui les lient étroitement aux humains.

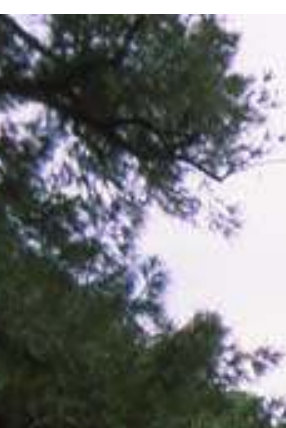

s
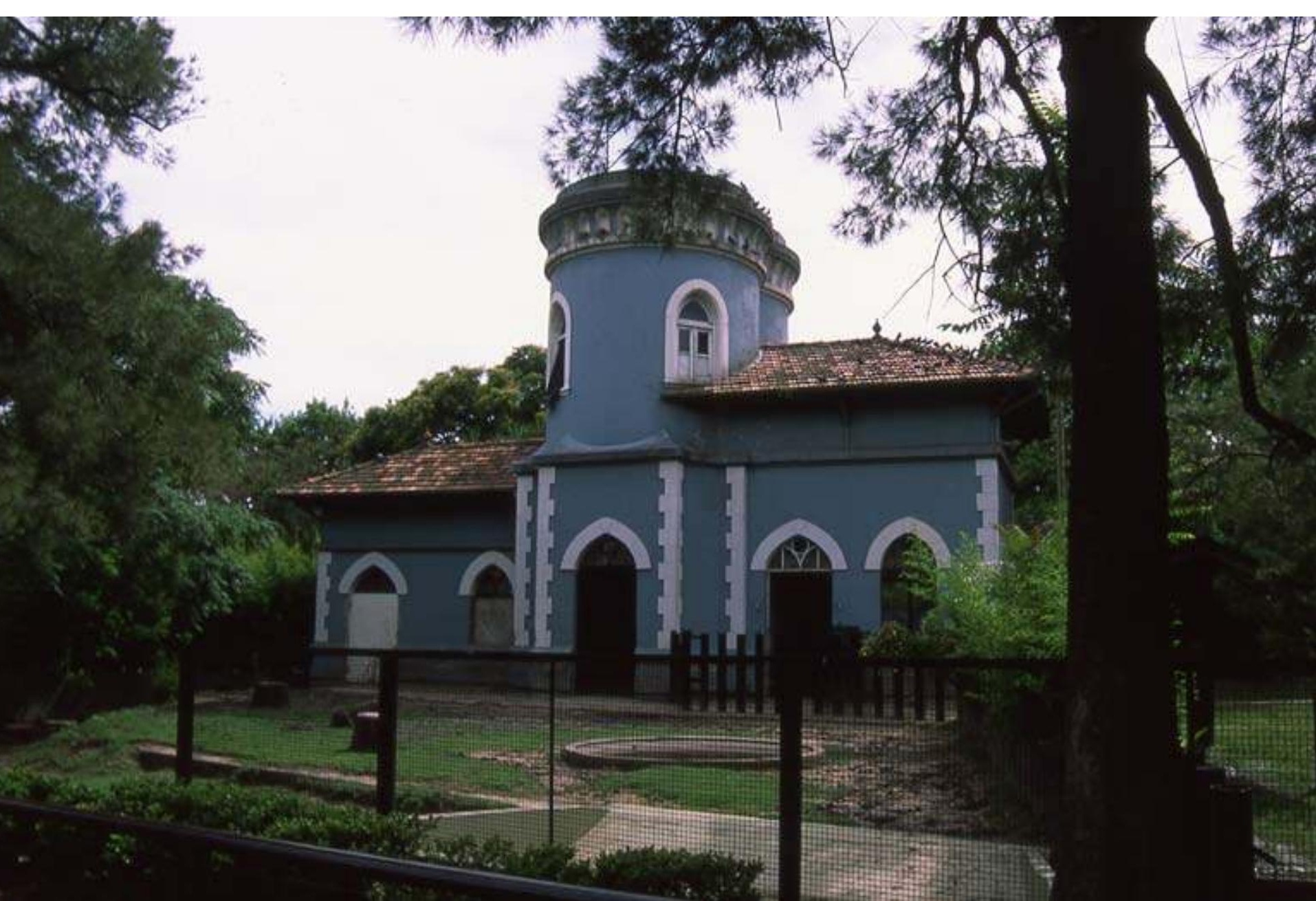


\section{Le thêâtre}

La configuration des espaces et l'environnement des zoos font partie des composantes essentielles qui entrent dans la construction de l'animal de zoo. Je ne peux m'attarder ici sur l'impact de l'évolution architecturale des zoos dans cette construction. Cependant, l'histoire de l'architecture des zoos et de la configuration des enclos révèle quelques aspects des motivations humaines et de l'imaginaire développé autour des animaux ${ }^{7}$. Je voudrais mettre l'accent sur la conception de l'architecture, de la configuration, de l'organisation et de la gestion des espaces et des lieux du zoo comme éléments d'un théâtre et d'une représentation. Selon Eric Baratay et Elisabeth Hardouin-Fugier, les zoos sont des « théâtres du monde sauvage » (2002: 13) dont les enclos forment un décor « théâtral [...] symbolisant les environnements plus qu'ils ne les reproduisent » (2002:139, mes italiques). Les directeurs de zoo et les architectes créent une pièce de théâtre sur le thème du monde sauvage au moyen de ces décors et des animaux/acteurs qui y interprètent un rôle. C'est sur un aspect précis que je voudrais m'arrêter: la manière dont s'y prend le zoo pour entraîner le visiteur dans l'ailleurs d'un monde sauvage fictif.

Jusqu'au début du $\mathrm{XX}^{\mathrm{e}}$ siècle probablement, la proximité immédiate des animaux « exotiques » exposés devait suffire à captiver la plupart des visiteurs de zoo, qui n'avaient jusque-là jamais rencontré d'animaux de ce genre - l'absence préalable de rencontre en chair et en os les rendait suffisamment intéressants à voir en eux-mêmes. Bien sûr, il y avait une référence à un ailleurs, à un autre univers, parce qu'ils étaient importés de régions lointaines et « exotiques », mais c'est l'animal inhabituel luimême qui était au centre de l'attention. À partir de l'époque de Carl Hagenbeck (et particulièrement de l'ouverture de son zoo à Stellingen, près de Hambourg, en 1907), les concepteurs et directeurs de zoo ont cherché à mettre en scène une histoire plus complexe avec des décors naturalistes, mais pour l'essentiel l'histoire se déroulait encore, semble-t-il, à l'intérieur du zoo. Comme il était peu probable que le visiteur moyen de la fin du XIX et du début du XX ${ }^{e}$ siècle ait eu l'occasion d'expérimenter le monde arctique ou les plaines d'Afrique de l'Est, ces régions étaient représentées au zoo à son intention - le meilleur des cadres pour apprécier les animaux exposés devant eux. Les animaux étaient importés (au sens littéral, car il n'y avait pas ou peu de reproductions dans les zoos à cette époque) de régions lointaines et exotiques, mais l'intérêt était davantage dans la confrontation directe à l'animal.

Pour nombre de zoos modernes, cette proximité immédiate et la simple présence des animaux ne suffisent plus. L'attention du visiteur est dirigée non vers l'intérieur du zoo lui-même, mais vers l'extérieur, vers les espaces naturels où les animaux devraient idéalement vivre et être vus ${ }^{8}$. Les zoos doivent désormais traiter de conservation, de biodiversité ou d'écologie liées à ces espaces, et, pour y parvenir, ils doivent créer dans leur propre espace des univers complexes. Les zoos modernes et bien 
dotés tentent de créer des aménagements qui déguisent et nient parfois l'origine géographique du zoo. L'idéal étant de faire oublier au visiteur qu'il se trouve à Zurich ou à San Diego, et de lui offrir la sensation et les conditions d'une forêt tropicale humide ou de la savane africaine. Cet univers doit être parfaitement reproduit dans sa totalité, si réel que le visiteur ne peut qu'y croire. Les zoos veulent attirer le public pour le transporter ailleurs par les sens et l'imagination. David Hancocks, un directeur de zoo parmi les plus créatifs du monde, a récemment écrit un livre intitulé non sans raison A Different Nature, qui décrit sa vision du zoo moderne (2001). Les visiteurs devraient être «immergés» dans les paysages des zoos - signifiant par là une immersion dans les paysages construits à l'intérieur des zoos, plutôt que dans le paysage de l'espace du zoo lui-même. Selon lui, «On s'efforce de plus en plus de recréer pour les visiteurs les conditions originales de l'habitat, plutôt que de se contenter d'exposer les animaux » (Hancocks 2001 : 118) ${ }^{9}$. Ici, ce sont les visiteurs qui sont privilégiés, la recréation d'habitats naturels dans les espaces d'exposition du zoo étant conçue pour eux plutôt que pour les animaux - bien que certains de ces environnements présentent, par leur complexité, des avantages pour ces derniers. Tout aussi « réelles » que soit les apparences de ces espaces, ce sont des illusions conçues pour enchanter les visiteurs, illusions dont ils sont les spectateurs ou les acteurs partiels. C'est le monde de l'« hyperréalité » de Baudrillard (1994) et d'Eco (1995), c'est-à-dire une simulation, voire un simulacre. C'est aussi le monde de la « mise en scène de l'authenticité » et du « pseudo-évènement », tels qu'analysés par Dean MacCannell (1999) dans l'univers du tourisme et dans le monde complexe du parc à thème postmoderne.

Ces univers de zoo ont l'apparence, l'odeur et les sons du monde réel, leur véracité est convaincante en surface, en tant qu'univers superficiels, mais bien entendu ils ne sont pas ce qu'ils prétendent. Ils ne sont pas le monde réel vécu par les homologues sauvages des animaux de zoo, dans lequel ils luttent et se battent. Les animaux de zoo jouent le rôle des animaux sauvages. Mais ce rôle est limité et l'expression de nombreux aspects de leur comportement naturel - en particulier ceux susceptibles de les compromettre en tant qu'animaux de zoo - ne leur est pas permise; ils ne peuvent être ni prédateur, ni proie. Toujours selon David Hancocks, « les zoos doivent rappeler à leurs visiteurs urbains le caractère sauvage des animaux sauvages... les zoos doivent trouver un moyen de convaincre les gens de la splendeur, de la beauté, de la rudesse et de la réalité du monde sauvage » (2001 : 119). Seulement ils ne peuvent présenter la « réalité » du monde sauvage dans les limites du zoo - tout au plus peuvent-ils en offrir une représentation. Les zoos peuvent être vus comme des théâtres d'inauthenticité (l'ici) essayant de raconter des histoires authentiques (l'ailleurs).

Sans nul doute, certains zoos modernes adoptent progressivement ce point de vue. Pour leurs directeurs, la diffusion des questions de conservation, de biodiversité et de prise de conscience environnementale constitue le seul objectif acceptable pour les zoos aujourd'hui, et la principale caution morale pour garder en captivité des animaux sauvages. Ce qui n'est pas chose 
aisée à réaliser, en raison même de la présence de l'animal utilisé comme support pour diffuser ces messages. Chaque animal doit signifier son espèce et la place de cette espèce dans la nature. Sur le plan éthique, le maintien en captivité d'animaux sauvages est jugé acceptable car il rend service à leurs homologues en liberté, et à la nature en général. Labsence de rencontre directe ou d'interaction entre eux et les visiteurs - qui pourraient altérer ou réduire l'« essence » de leur caractère sauvage - permet de les percevoir, de les imaginer et de les représenter comme des animaux sauvages.

\section{Les spectateurs}

Gorille

Derrière la vitre... Zooparc de Beauval, 2002.
Mais il ne faut pas négliger la possibilité d'autres types de rencontres entre l'homme et l'animal de zoo. Certains animaux de zoo se déplacent encore du sauvage vers le domestique et sont apprivoisés afin de montrer au public, en dehors de leurs enclos, les habiletés et les comportements qu'ils produiraient dans la nature. Ces spectacles ont pour but d'éduquer le public et de le divertir avec une démonstration de virtuosité animale telle qu'elle aurait été nécessaire dans la nature. Dans ces spectacles, souvent présentés comme "une expérience de l'univers de la faune ", les animaux apprivoisés sautent, manipulent des objets, nagent, bondissent ou descendent en piqué sur des cibles. Mais ces actions n'ont rien d'authentiques. Elles n'ont d'autre objectif que la performance elle-même: l'animal de zoo joue le rôle de l'animal sauvage ${ }^{10}$.

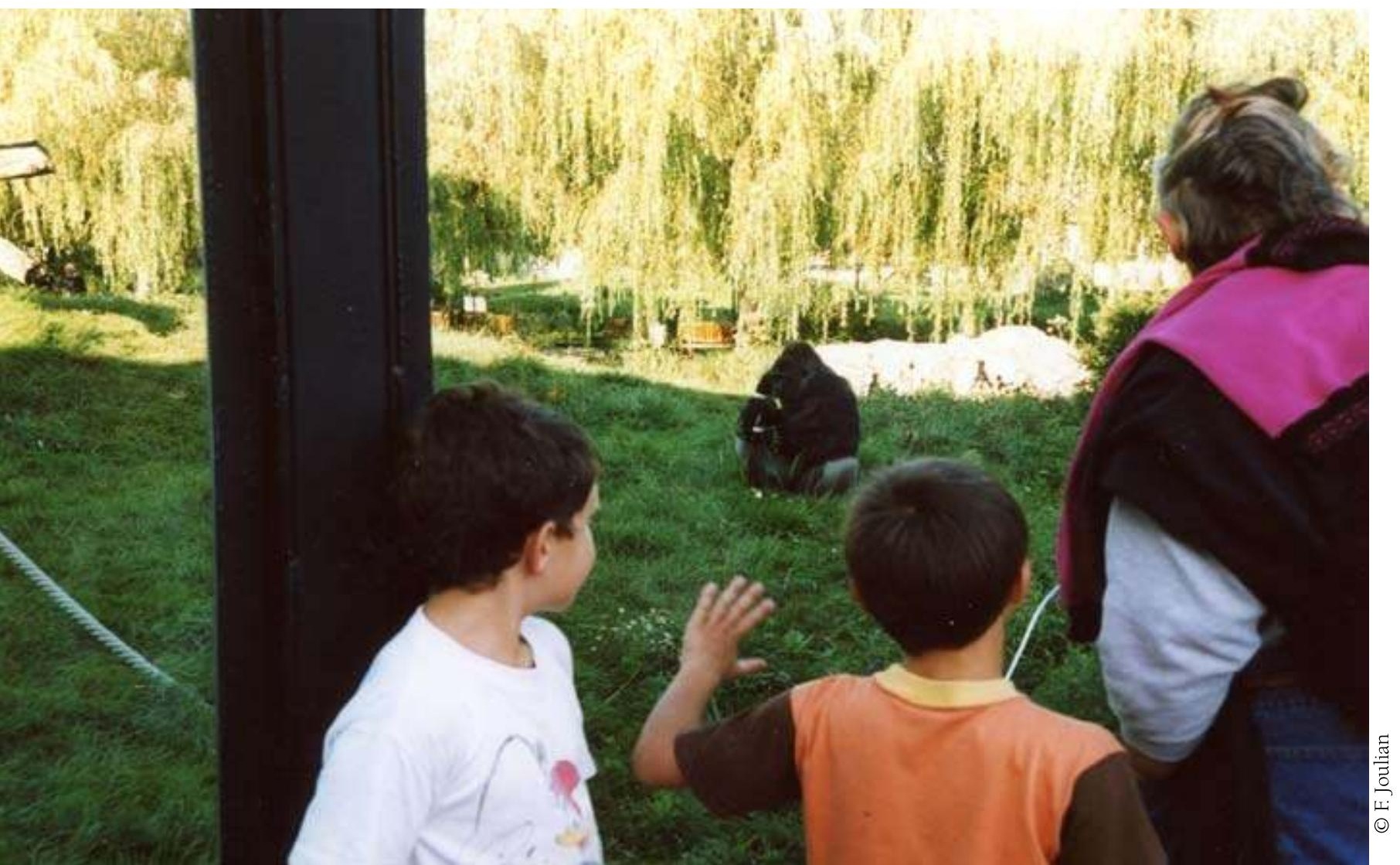


D'autres types de performances et d'autres interactions, individuelles, informelles et non scénarisées deviennent plus problématiques car elles brisent le charme, mettent en question l'identité des animaux en tant qu'animaux sauvages et attirent l'attention sur l'immédiateté de l'animal en tant qu'animal de zoo. Les gens vont au zoo car ils savent qu'ils y trouveront ces animaux et savent qu'ils seront divertis. Si les directeurs de zoos veulent les mener vers d'autres univers et d'autres contrées, la majorité des visiteurs en revanche ne semblent pas vouloir se laisser embarquer ${ }^{11}$. C'est cet animal, particulier, individuel, dans l'enclos, qui leur importe, c'est cet animal, particulier, individuel, qui peut devenir une source d'intérêt, de divertissement et d'excitation, non une qualité abstraite ou un ensemble de relations qui lui sont associées. Ils aiment le voir faire quelque chose - manger, se battre, copuler, quémander de la nourriture -, le voir jouer un rôle pour eux. Peut-être le phénomène est-il mieux formulé ainsi : le comportement des animaux est commenté et interprété comme une performance significative sur le plan humain (« ces deux--là sont des amis », " elle ne l'aime pas », " celui-ci est fainéant», " il te fait coucou », " elle sourit », " le moche me fait penser à oncle Jacques »). Les visiteurs ne se contentent pas de répondre aux animaux de cette manière personnelle et anthropomorphique, ils viennent également avec toute une cohorte d'images, d'idées et de représentations. La plupart d'entre elles ne sont pas liées à la conservation, à l'écologie ou à la biodiversité. Ces histoires, ces images et ces représentations puisent dans les dessins animés, les livres d'enfants, les films, et sont ensuite projetées sur les animaux qui leur font face. Nombre de visiteurs construisent les animaux des zoos d'après ces images, qui divergent des images éthologiques et environnementales des professionnels du zoo.

Ce qui, à présent, m'intéresse particulièrement à propos des relations homme/animal - en cette modernité tardive, cette postmodernité ou toute autre définition du présent -, c'est la variabilité des relations entretenues par différentes catégories de personnes avec des animaux sauvages. Je crois que l'on perçoit une tendance à l'augmentation de rencontres avec ces derniers. Dans le monde occidental, l'appétit pour les documentaires d'histoire naturelle télévisés est apparemment insatiable, la part de l'écotourisme dans l'industrie touristique en général ne cesse de croître, les gens dépensent des fortunes pour faire des safaris un peu partout dans le monde, ils prennent la mer pour observer les baleines et les dauphins, tandis que les parcs à thème animalier semblent gagner en popularité.

Toutes ces pratiques donnent accès à ces animaux considérés comme sauvages et authentiques, vivant dans leur milieu naturel. Mais elles ne freinent pas pour autant l'élan de millions de personnes à travers le monde, qui continuent à visiter les zoos. Bien que l'on puisse considérer les animaux de zoo comme des pâles représentations de leurs homologues sauvages vivant dans une pâle représentation de leur habitat naturel, les gens ont toujours le désir de les voir et de les rencontrer dans ce cadre-là. En un sens, les animaux sauvages qui habitent les zoos sont des créatures déplacées, vivant dans un environnement étranger, mais pourtant, et 
cela est extrêmement important, c'est de là qu'ils viennent. Ceux qui sont nés au zoo ne sont pas déplacés, il s'agit là de leur maison, ils n'en ont jamais connue d'autre. Les animaux de zoo ne souffrent pas de la nostalgie d'une autre époque ou d'un autre lieu. Du point de vue des visiteurs également, le zoo peut être considéré comme une "maison » pour animaux sauvages: c'est là qu'ils s'attendent à rencontrer des lions, des éléphants, des girafes et des zèbres dans leur ville.

Les modes de représentation des animaux sauvages par leurs homologues de zoo ont changé historiquement et varié culturellement, en particulier sur le plan du style d'exposition. David Hancocks a soutenu que les modes d'exposition des animaux sauvages ont conduit les zoos à en perpétuer de «fausses images».

«La manière dont les zoos présentent les animaux aux gens forge les opinions populaires à propos des animaux sauvages. Leurs dispositifs façonnent littéralement le point de vue du public. Souvent les visiteurs ne perçoivent pas les animaux de zoo comme des animaux sauvages parce qu'ils ne sont pas présentés en tant que tels» (Hancocks 2001 : 149).

Pour lui, les enclos devraient véhiculer l'idée de l'habitat naturel des animaux afin que les animaux qui s'y trouvent évoquent leurs homologues sauvages. Il semble que la simple présence corporelle de l'animal n'y suffise pas. Seul, il ne peut être un animal sauvage; il peut seulement en être une image fausse ou appauvrie. De ce point de vue, le décor, l'arrangement spatial de l'exposition, est d'une certaine manière plus important que le corps animal lui-même. Le raffinement toujours croissant des technologies de la conception et de la construction donne lieu à la création de mondes sauvages hyperréels, mais toujours artificiels. Dans ces mondes, l'animal de zoo doit jouer le rôle de l'animal sauvage avec davantage d'efficacité et de conviction. Ces approches globales permettent plus efficacement aux zoos d'évoquer l'ailleurs et le non-présent tout en restant à leur place et dans leur présent. Des premières cages prisons aux enclos sans barrières et jusqu'à la plupart des reconstitutions d'habitat réalistes, les animaux de zoo n'ont jamais simplement été des animaux présents pour eux-mêmes, mais représentant. Ils ont toujours été et restent le produit de l'imaginaire que l'homme développe autour du monde sauvage et de ses rapports aux sociétés et cultures humaines.

Traduction Vincent Leblan 


\section{NOTES}

1. Certaines des idées présentées dans cet article sont parues sous une version plus générale dans un essai auquel j'ai contribué (Billingham 2007). Je remercie les éditeurs de m'avoir permis d'en utiliser une partie pour cet article.

2. Ce point mérite d'être nuancé. Les zoos contiennent effectivement des animaux considérés comme « domestiques », par exemple ceux que l'on rencontre dans les espaces étudiés spécifiquement pour les enfants. Ou encore, les zoos d'un pays donné peuvent contenir des animaux « sauvages » qui sont perçus comme domestiqués ou « semidomestiqués » dans un autre pays. Par exemple dans un zoo européen, un lama serait représenté comme un animal sauvage, alors qu'au Pérou il s'agit d'une bête de somme domestique.

3. Deux articles récents de Ralph Acampora (2001a, $2001 \mathrm{~b}$ ) adoptent un point de vue critique sur les problèmes du constructivisme social/culturel et guident le lecteur à l'intérieur de l'abondante littérature dans ce domaine.

4. Pour une discussion récente de la complexité des problèmes relatifs à la domestication, voir Cassidy \& Mullin 2007.

5. De ce point de vue, l'existence de stud-books internationaux pour planifier la reproduction de nombreuses espèces menacées n'est pas sans rappeler le suivi du pedigree des animaux domestiques.

6. Cette affirmation doit cependant être nuancée. Nombre d'animaux dans les zoos - certains reptiles ou les poissons peut-être - peuvent ne pas percevoir le regard humain.

7. Pour un compte rendu récent et détaillé de l'histoire de la configuration et de l'architecture des zoos, voir, Baratay \& Hardouin-Fugier (2002). Pour un compte rendu plus virulent et critique de l'architecture des zoos modernes, voir Hancocks (2001) et voir Mullan \& Marvin (1999) pour des éléments de débat issus de mes recherches précédentes.

8. La philosophie, la conception et les motivations des principes d'exposition de nombreux zoos modernes résultent de la rencontre de multiples processus sociaux et culturels qui ne peuvent être abordés ici. Il faudrait compter avec l'essor du documentaire d'histoire naturelle qui a fait connaître la vie de la plupart des animaux à une large audience, la préoccupation pour le bien-être animal et le développement de la conscience écologique, ainsi que la création d'espaces de loisirs et de divertissements complexes et élaborés tels que les parcs à thème.

9. Hancocks est très conscient des problèmes concernant le bien-être animal dans les zoos et ne cesse de souligner la nécessité d'adapter les espaces d'exposition aux animaux qui y sont placés. Je ne puis traiter cet aspect de l'exposition mais je souhaite reconnaître l'importance des contributions de Hancocks ici.

10. Ces performances et ces formes d'exposition risquent, par leur interprétation et les réactions du public, de rapprocher l'image de l'animal de zoo d'une autre catégorie intermédiaire entre le sauvage et le domestique - celle de l'animal de cirque. Les zoos modernes occidentaux cherchent à l'éviter. Voir Paul Bouissac (1972) pour une interprétation des contrastes de la distance et du contact dans les zoos et les cirques.

11. Voir par exemple le travail de Stephen Kellert $(1979,1984,1989)$ sur la compréhension et la connaissance des visiteurs sur les animaux après la visite des zoos. 


\section{RÉFERENCES}

Acampora, Ralph, 2001a, "Real Animals? An Inquiry on Behalf of Relational Zoöntology" Human Ecology Review 8 (2): 73-78.

Acampora, Ralph, 2001 b "Representations Cubed : Reviewing Reflections on Animal Imagery", Society and Animals 9 (3) : 299-307.

Baker, Steve, 2001, "Animals, Representation, and Reality" Society and Animals 9 (3): 189-201.

Baratay, Eric \& Hardouin-Fugier, Elisabeth, 2002, Zoo : a History of Zoological Gardens in the West. London, Reaktion Books.

Baudrillard, Jean, 1994, Simulacra and Simulations. Ann Arbor, University of Michigan Press.

Bertens, Hans, 1995, The Idea of the Postmodern: a History. New York, Routledge.

Billingham, Richard, 2007, Zoo. Birmingham, Vivid.

Bouissac, Paul, 1972, "A Study of 'Wild' Animal Displays in Circuses and Zoos", Journal of Popular Culture VI(3) : 607-614

Cassidy, Rebecca and Mullin, Molly, 2007, Where the Wild Things are Now: Domestication Reconsidered. Oxford, Berg.

Eco, Umberto, 1995, Faith in Fakes: Travels in Hypereality. London, Minerva.

Hancocks, David, 2001, A Different Nature: The Paradoxical World of Zoos and Their Uncertain Future. Berkeley, University of California Press.

Kellert, Stephen, 1979, "Zoological Parks in American Society", Proceedings of the American Association of Zoological Gardens and Aquariums: 1-39

Kellert, Stephen, 1984, "Urban Perceptions of Animals and the Natural Environment", Urban Ecology 8: 209-28.

Kellert, Stephen \& Dunlap, Julie, 1989, "Informal Learning at the Zoo", Report to the Zoological Society of Philadelphia, Philadelphia, ZSP.

MacCannell, Dean, 1999, The Tourist. Berkeley, University of California Press.

Marvin, Garry, 2001, "Cultured Killers: creating and representing foxhounds", Society and Animals 9 (3): 273-292.

Mullan, Bob \& Marvin, Garry, 1999, Zoo Culture. Urbana, University of Illinois Press. 


\section{RÉSUMÉ}

L'animal de zoo. Un rôle entre sauvage et domestique. Cet article s'interroge sur ce qui caractérise les animaux sauvages montrés au public dans les zoos. Il semble que ces animaux peuvent être perçus comme des créatures mi-sauvages, mi-domestiques, et qu'ils partagent vraisemblablement la même identité: celle de l'animal de zoo. Partant du constat que leur mode de vie est, en apparence, artificiel, cet article propose également une réflexion sur leur statut d'acteurs auxquels incombe le rôle - certes limité - de représenter leurs congénères sauvages. Les zoos sont ainsi envisagés ici comme un décor de théâtre destiné à la production de discours et d'imaginaire sur le monde sauvage.

\section{ABSTRACT}

Configuring the Zoo Animal: A Role Between the Wild and the Domesticated. This article explores some issues relating to the nature of wild animals kept for public exhibition in zoos. It suggests that such animals can be interpreted as being creatures which are between the wild and the domesticated and that they perhaps share an identity - that of "zoo animal". The article also considers how such animals, living apparently inauthentic lives in zoos, can be considered as actors performing the role (albeit limited and restricted) of representing their wild counterparts. Zoos are also considered here as theatrical settings for telling stories about natural worlds that exist beyond their boundaries.

\section{MOTS CLÉS}

Zoo, représentation, animal sauvage, exposition.

\section{KEYWORDS}

Zoo, representation, wild animal, exhibition. 